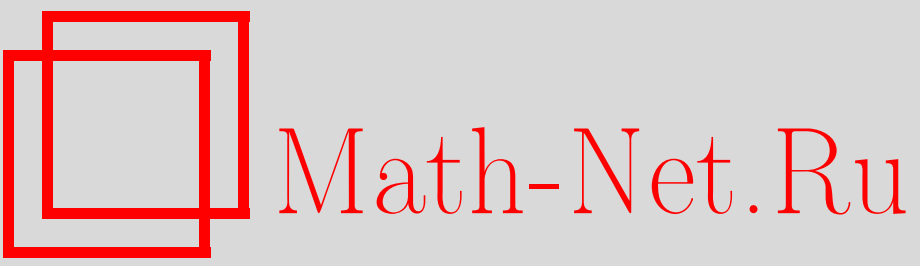

Я. Б. Воробец, Әргодичность бильярдов в многоугольниках: явные примеры, УМН, 1996, том 51, выпуск 4, 151-152

DOI: https://doi.org/10.4213/rm999

Использование Общероссийского математического портала Math-Net.Ru подразумевает, что вы прочитали и согласны с пользовательским соглашением

http://www.mathnet.ru/rus/agreement

Параметры загрузки:

IP: 3.85 .73 .92

26 апреля 2023 г., 05:27:59 


\title{
ЭРГОДИЧНОСТЬ БИЛЬЯРДОВ В МНОГОУГОЛЬНИКАХ: ЯВНЫЕ ПРИМЕРЫ
}

\author{
Я. Б. ВоРОБЕЦ
}

Бильярдом в плоской области $Q$ называется динамическая система, описывающая движение в $Q$ без трения точечного шарика, отражающегося от границы $Q$ по закону "угол падения равен углу отражения". Бильярдный поток обычно рассматривается на поверхности уровня энергии, соответствующей движению с единичной скоростью. В таким образом выбранном фазовом пространстве $Q \times S^{1}$ ( $S^{1}$ - окружность единичных скоростей) имеется естественная мера, которая инвариантна относительно потока.

В настоящей работе рассматриваются билярдные потоки в многоугольых областях общего вида. Для таких областей условие отражения бильярдного шара от границы особенно просто, однако наличие угловых точек приводит к тому, что, за несколькими исключениями, бильярд в многоугольнике является динамической системой с особенностями. Ввиду этого изучение эргодических свойств потока оказывается сложной задачей. Общие результаты здесь получены лишь для бильярдов в рациональных многоугольниках.

ОПРЕДЕЛЕНИЕ 1. Многоугольник $Q$ называется рациональным, если группа $G(Q)$, порожденная линейными частями отражений относительно сторон $Q$, конечна. Эквивалентное условие - угол между любыми двумя сторонами многоугольника (не обязательно соседними или принадлежащими одной компоненте границы) рационально соизмерим с $\pi$.

Для рационального многоугольника $Q$ фазовое пространство бильярда расслаивается на инвариантные поверхности вида $Q \times G(Q) v, v \in S^{1}$ (см. [5]). Как показали Керкгоф, Мазур и Смайли [1], сужение билярдного потока на почти каждую из этих поверхностей строго эргодично, т.е. имеет единственную инвариантную нормированную борелевскую меру. В частности, указанные поверхности являются эргодическими компонентами потока. Исходя из этого результата, с помощью аппроксимационных методов было показано (см. [1], [2]), что в пространстве всех многоугольников, а также в тех его замкнутых подпространствах без изолированных точек, где плотны рациональные многоугольники, найдется остаточное множество, состоящее из многоугольников, бильярд в которых эргодичен (т.е. в фазовом пространстве бильярда любое измеримое инвариантное подмножество имеет нулевую или полную меру).

А.М. Стёпин предложил автору попытаться конструктивизировать доказательства сформулированных выше результатов с тем, чтобы получить конкретные примеры многоугольников с эргодическими бильярдными потоками. Результатом таких попыток явилась приводимая ниже теорема 1.

ОПРЕДЕЛЕНИЕ 2. Пусть $\delta(N)$ - убывающая к нулю положительная функция натурального аргумента. Скажем, что многоуголник $Q$ допускает аппроксимацию рациональнылми многоугольниками со скоростью $\delta(N)$, если найдутся сколь угодно большие номера $N$ с тем свойством, что попарные углы $\alpha_{1}, \alpha_{2}, \ldots, \alpha_{k}$ между сторонами многоугольника $Q$ с точностью $\delta(N)$ аппроксимируются углами вида $\pi n / N$, где $n$ - целое число, причем дроби $n_{1} / N, n_{2} / N, \ldots, n_{k} / N$, соответствующие разным углам, нельзя сократить на одно и то же число.

ТеОрема 1. Если многоугольник $Q$ допускает аппроксимацию рациональными многоугольниками со скоростью

$$
\delta(N)=\left({ }_{2} 2^{2^{2}}\right)^{-1}
$$

то бильярдный поток в $Q$ әргодичен.

Например, эргодичным будет бильярдный поток в прямоугольном треугольнике с острым углом $\pi\left(a_{5}^{-1}+a_{10}^{-1}+\cdots+a_{5 n}^{-1}+\cdots\right)$, где $\left\{a_{n}\right\}-$ последовательность, заданная соотношениями $a_{0}=1, a_{n+1}=2^{a_{n}}$ при $n=0,1,2, \ldots$.

Остановимся вкратце на основных идеях доказательства теоремы. Оно состоит из двух частей: во-первых, нужно провести конструктивные оценки, касающиеся аппроксимации многоугольников и бильярдных потоков в них - это сравнительно несложно; во-вторых, нужно получить конструктивньй вариант эргодической теоремы для бильярдного потока в рациональном 
многоугольнике - и здесь заключена главная трудность. Доказательство из [1], в особенности используемые там методы теории Тайхмюллера, не оставляет никаких надежд на конструктивизацию. Поэтому в качестве отправной точки было избрано другое доказательство того же факта, полученное Бошерницаном [3] и Мазуром [4]. Конечные результаты излагаются ниже.

Пусть $Q$ - рациональный многоугольник, $\alpha(Q)$ - сумма всех его внутренних углов, $s(Q)-$ длина кратчайшей обобщенной диагонали (т.е. билярдной траектории с концами в вершинах $Q$ ). Положим также $m(Q)=\frac{|G(Q)| \cdot \alpha(Q)}{2 \pi}(m(Q)$ - целое число), $S(Q)=|G(Q)| \cdot \operatorname{Area}(Q)$. Для любой измеримой функции $F(x, v)$ на $Q \times S^{1}$ обозначим через $S^{t} F(x, v)$ ее среднее значение за время $t$ под действием бильярдного потока в $Q$, через $F_{0}(x, v)$ - предел в смысле сходимости почти всюду функций $S^{t} F(x, v)$ при $t \rightarrow \infty$ :

$$
F_{0}(x, v)=\frac{1}{S(Q)} \sum_{g \in G(Q)} \int_{Q} F(y, g v) d y .
$$

Теорема 2. Пусть $L, E>0$. Предположим, что для любого направления $v \in S^{1}$ функция $f_{v}(x)=F(x, v)$ является липшицевой функцией на $Q$ с постоянной Липшица $L$; пусть, кроме того, $F(x, v)=0$ при $x \in \partial Q u|F(x, v)| \leqslant E$ для всех $x \in Q, v \in S^{1}$. Тогда для любого $\varepsilon, 0<\varepsilon \leqslant 0.999$, имеем:

$$
\begin{gathered}
\frac{1}{2 \pi \text { Area }(Q)} \int_{Q \times S^{1}}\left|S^{t} F(x, v)-F_{0}(x, v)\right| d \mu(x) d \lambda(v) \leqslant 4 E \varepsilon \\
n p u \quad t \geqslant \frac{L \cdot S(Q)}{E}\left(\frac{1}{\varepsilon}\right)\left(H(m(Q)) \cdot S(Q) / s^{2}(Q) \cdot 1 / \varepsilon\right)^{m(Q)+5}
\end{gathered}
$$

əде $H(1)=2^{60}, H(m)=(500 m)^{(2 m)^{2 m}}$ nри $m>1, \mu-м е р а$ Лебега на $Q, \lambda-$ мера Лебега на $S^{1}$, нормированная так, что $\lambda\left(S^{1}\right)=2 \pi$.

Все (кроме двух особых) инвариантные поверхности $Q \times G(Q) v$ в фазовом пространстве бильярда в $Q$ гомеоморфны некоторой поверхности $M$ и согласованным образом задают на $M$ плоскую структуру - метрику нулевой кривизны с конечным числом особых точек, в каждой из которых имеется коническая особенность с углом вида $2 \pi m$, где $m$ - целое число (кратность особой точки). Для доказательства теоремы 2 важно иметь оценку величины $N(L)$ - числа седлосвязок (геодезических отрезков, соединяющих особые точки) на поверхности $M$, длина которых не больше $L$ (ср. [3]). Оценка в форме $N(L)=O\left(L^{2}\right)$ при $L \rightarrow \infty$ была доказана Мазуром [4]. Конструктивная оценка, полученная автором, такова.

Теорема 3. Пусть $m$ - сумма кратностей особых почек плоской структуры, $s$ длина кратчайшей седлосвязки. Тогда

$$
N(L) \leqslant h(m) \cdot(L / s)^{2},
$$

где $h(1)=\left(3 \cdot 2^{7}\right)^{6}, h(m)=(400 m)^{(2 m)^{2 m}}$ npu $m>1$.

Автор благодарен проф. А.М. Стёпину за постановку задачи и постоянную поддержку в течение работы.

\section{СПИСОК ЛИТЕРАТУРЫ}

[1] Kerckhoff S., Masur H., Smillie J. // Ann. of Math. 1986. V. 124. № 2. P. 293-311. [2] Pospiech F., Stepin A. M. // Wiss. Z. Univ. Halle. 1988. V. 37. № 4. P. 89-98. [3] Boshernitzan M. // Duke Math. J. 1985. V. 52. № 3. P. 723-752. [4] Masur H. // Ergod. Th. \& Dynam. Sys. 1990. V. 10. № 1. Р. 151-176. [5] Земляков А. Н., Каток А. Б. // Матем. заметки. 1975. T. 18. № 2. C. 291-300.

Московский государственный 